\title{
Active positioning device for a perimodiolar cochlear electrode array
}

\author{
B. Y. Arcand, P. T. Bhatti, N. V. Butala, J. Wang, C. R. Friedrich, K. D. Wise
}

Abstract The authors report on an actuated positioning device for a cochlear prosthesis insertion procedure. The device consists of multiple high-aspect ratio fluidic actuator chambers integrated with a high-density silicon cochlear electrode array and manufactured in a taperedhelix form. Actuation chambers with cross-sectional sizes as small as $40 \times 200 \mu \mathrm{m}$ and lengths of $30 \mathrm{~mm}$ have been fabricated using flexible polymers. The device will allow for low-resistance basilar insertion of a stimulating electrode array into the cochlea providing for deep, perimodiolar position considered most beneficial for auditory nerve stimulation, while minimizing intracochlear trauma. Experimental measurements, FEA analysis, and modeling demonstrate a viable and appropriate actuation method for a cochlear implant procedure.

\section{1}

\section{Introduction}

Cochlear implants have become an accepted and successful treatment for profound sensorineural deafness in both children and adults. Over 70,000 recipients of implants worldwide have experienced restoration of useful levels of auditory function. The wide spread use of multichannel devices in recent years has contributed greatly to the performance experienced by users of cochlear prostheses.

The cochlea is a snail-shaped, auditory organ responsible for converting the mechanical energy of sound waves to an electrical signal that can be interpreted by the brain. Sound received by the ear, is transferred by the small bones of the middle ear into the fluid filled spaces of the cochlea. Within the cochlea, the basilar membrane divides

Received: 8 August 2003 / Accepted: 16 November 2003

B. Y. Arcand ( $\varangle)$, N. V. Butala, C. R. Friedrich NSF Engineering Research Center for Wireless Integrated Microsystems, Department of Mechanical Engineering, Engineering Mechanics, Michigan Technological University, 1400 Townsend Drive, Houghton, MI 49931, USA e-mail: byarcand@mtu.edu

P. T. Bhatti, J. Wang, K. D. Wise

NSF Engineering Research Center for Wireless Integrated Microsystems, University of Michigan,

Michigan, USA

This work is supported in part by the Engineering Research Centers Program of the National Science Foundation under Award Number EEC-9986866. The authors would also like to thank Dymax for the UV cure adhesive samples they provided. the cochlea into separate chambers (Fig. 1) and has mechanical properties that tune its response to different frequency levels along its spiral length. This allows the cochlea to have sensitivity to high frequency sound towards the larger basilar end and low frequencies at the smaller apex. The deflection of the basilar membrane stimulates hair cells located in the adjacent organ of Corti, which in turn transmit an electrical signal through the spiral ganglion cells located in the cochlear modiolus to the auditory nerves responsible for sound perception. Trauma, degeneration due to disease, and deformities all can disrupt the delicate mechanisms of sound transduction within the cochlea and cause profound deafness.

Typically, residual nerve fibers survive in the dysfunctional cochlea making electrical stimulation of the auditory nerves possible by means of a cochlear prosthesis. Cochlear prostheses have three main components. The first is the external microphone, which picks up sound impinging on the user. The sound is then processed in a signal analyzer which filters the sound and maps the signal to corresponding electrodes on the implant. Finally, current is driven from the processor to the electrodes on the implant within the cochlea, stimulating nearby nerve fibers. Due to the tonotopic organization of the cochlea, stimulation of localized groups of nerves responsible for discrete frequency ranges is possible. In this way, a cochlear implant with multiple channels and a high-density electrode array can divide the sound signal into frequency bands and stimulate auditory nerves of the corresponding frequency range. The result is a higher degree of frequency resolution and multiple levels of sound perception, which translates into improved speech recognition for the implant recipient.

In addition to more frequency channels and a greater number of electrodes, positioning of the electrodes is crucial to the performance of the implant [1]. Close proximity of the electrode sites to the central nerve-bearing modiolus is essential for increasing spatial selectivity of stimulating electrodes. Not only does this allow for greater frequency resolution and dynamic range perceived by nerve subpopulations, but it also greatly reduces power threshold requirements [2-3].

Reduced power requirements improve the battery life (and size) for the prosthesis and also reduce the current densities at the electrode surfaces. High current densities are associated with the creation of harmful bi-products through electrochemical processes [4]. These harmful products could potentially lead to further irreversible deterioration of the cochlea. Stray currents that cause 


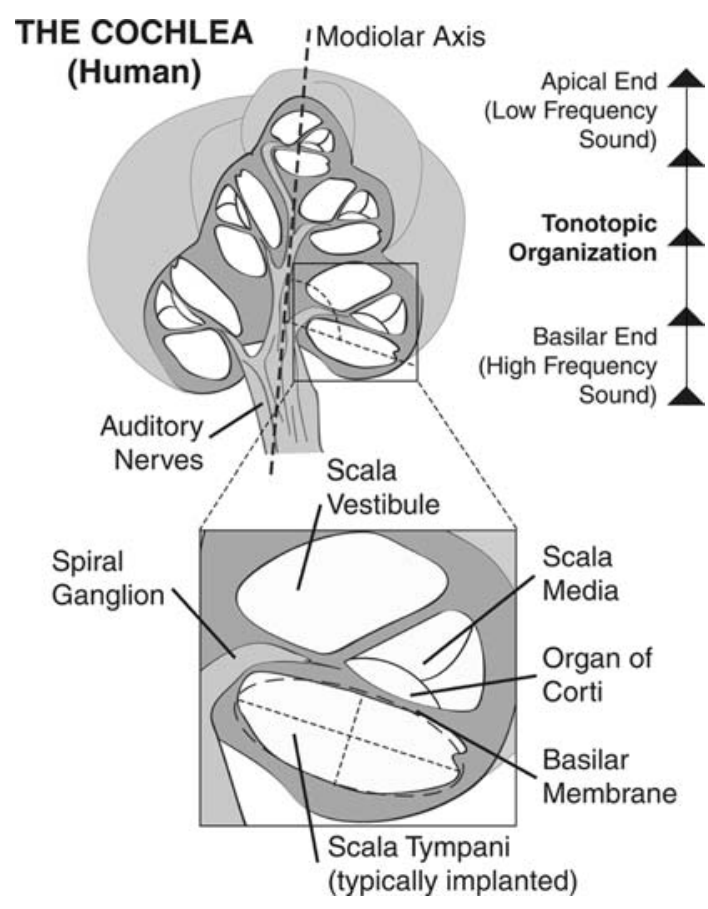

Fig. 1. Anatomy of the cochlea

unwelcome stimulations of facial muscles are also reduced with lowered thresholds.

To gain access to as many auditory nerves as possibly and the full spectrum of human sound perception, electrodes that are 'deeply' placed within the cochlea are needed. Implants are most often inserted in the basilar end of the cochlea, either through the round window or through a cochleostomy. To reach the apical end of the cochlea, and the nerves responsible for low frequency sound, the implant must navigate the long, narrow, helicalshaped corridor of the scala tympani. Implants in use today typically employ electrodes embedded in a flexible probe-like structure. Upon insertion, they use the internal walls of the cochlea to help guide themselves deeper within the scala tympani. This method can cause damage to the delicate internal structures of the cochlea promoting nerve degeneration and further loss of hearing potential in a patient [5]. Friction between the implant and the walls impedes deep insertion towards the apex. Insertions of these devices typically achieve less than half $\left(360-400^{\circ}\right)$ of the angular depth of the cochlea possible.

Moreover, by using the internal structures of the cochlea for insertion, the probe has a tendency to migrate towards the outer wall away from the nerve fibers. To overcome this, pre-curled implants have been developed that use additional stiffening structures to hold them straight for the initial stages of the insertion. This can complicate the insertion procedure in that the probe's additional stiffness can more easily cause damage while the curvature upon release of the stiffening structure is not easily controlled. While the new models have improved proximity of the electrodes to the modiolus, further improvements are in order.

Improvements to the insertion procedure can be realized by employing an implant that actively changes shape to fit the cochlea. By using fluidic actuators along the length of the implant, the shape can be changed to fit that of the cochlea during the insertion procedure. This would reduce wall contact and friction allowing for a deeper insertion while also avoiding damage to delicate internal structures. In addition, the implant can have a complex tapered-helical shape to closely 'hug' the modiolus when the insertion is completed. Here, a device to achieve actuation of a cochlear electrode array to provide deep perimodiolar placement within the scala tympani is presented.

\section{2}

\section{Conceptual design}

\section{Actuator principles}

The basic design of the fluidic actuators involves long flexible chambers with a high-aspect-ratio cross-sectional shape (Fig. 2). The chamber has a radius of curvature along its length with the minor axis of the cross-section being parallel to the radius. Radial actuation occurs when fluidic pressure is developed inside the chamber causing the cross-section shape to change. The response of the cross-section to the pressure is a tendency towards becoming more circular, the minor axis of the cross-section lengthens while the major axis decreases. The change in cross-section shape alters the bending characteristics of the chamber causing the overall radius to adjust in response, effecting actuation. The principle behind this actuation can be clearly seen in the mechanics formula for a curved beam [6]:

$\frac{1}{R}=\frac{M}{E I}$,

where a change in the cross-section shape corresponds to a change in the second moment of area $I$, while the quantities for elastic modulus $(E)$ and bending moment $(M)$ remain constant. $R$ is the bending radius of the structure and for the cochlear insertion tool is hear after called the curling radius. The bending moment can be thought of as a residual, built-in moment and since it and the elastic modulus are constant, the overall radius is proportional to the second moment of area. Therefore, when the minor axis of the cross-section is parallel to the overall curling radius, the application of pressure inside the chamber causes an increase in the curling overall radius of the chamber. The result is analogous to a highly elastic, large deformation, variable cross-section Bourdon-tube.

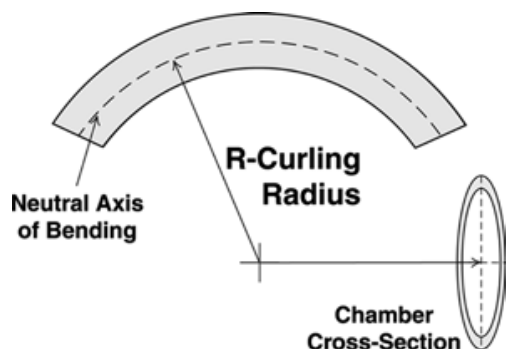

Fig. 2. Configuration of fluidic actuators. The minor axis of the cross-section is parallel to the curling radius 
This type of actuation is ideal for a cochlear implant since we would like the device to have a shape to closely hug the modiolus, but the radius of that spiral shape needs to temporarily be increased to allow for surgical insertion. Moreover, the change in radius can be precisely controlled with a corresponding change in fluidic pressure allowing for fine adjustments to closely fit the cochlea throughout the insertion procedure.

\section{Dimensional considerations}

The size and form of the actuation device was developed from an in-depth geometrical study of the guinea pig cochlea (Fig. 3) [7]. The guinea pig cochlea is approximately one-third the size of its human counterpart with an extra turn in the spiral helix. The average cross-section diameter of the scala tympani ranges from $1.26 \mathrm{~mm}$ at the basilar end to $0.28 \mathrm{~mm}$ at the apex. The length from the basilar entry point to the apex of the cochlea along the spiral centerline of the scala tympani is $14.63 \mathrm{~mm}$ and covers approximately $1358^{\circ}$ of angular extent. The curling radius of the spiral at the base is $1.48 \mathrm{~mm}$ and decreases to $0.20 \mathrm{~mm}$. The height of the spiral is $3.20 \mathrm{~mm}$.

\section{Device structure}

The design of the chambers not only accommodates the complex structure of the cochlea but also an attached monolithic silicon electrode array. The probe currently features 32 iridium-stimulating sites on a $250 \mu \mathrm{m}$ pitch and will eventually incorporate 128 sites. The inserted portion of the array has a $4 \mu \mathrm{m}$ thick substrate while the thicker $(14 \mu \mathrm{m})$ portion remains outside of the cochlea to support electrical connections. The array tapers from a width of $200 \mu \mathrm{m}$ at the tip (apical end) to $620 \mu \mathrm{m}$ at the basil end over a $10 \mathrm{~mm}$ length. Since the guinea pig cochlea has an additional spiral turn when compared to the human cochlea, research has focused on developing the tool for the first $10 \mathrm{~mm}$ of the insertion depth (this length encompasses the same number of turns as the human cochlea). Within the scala tympani, the array will

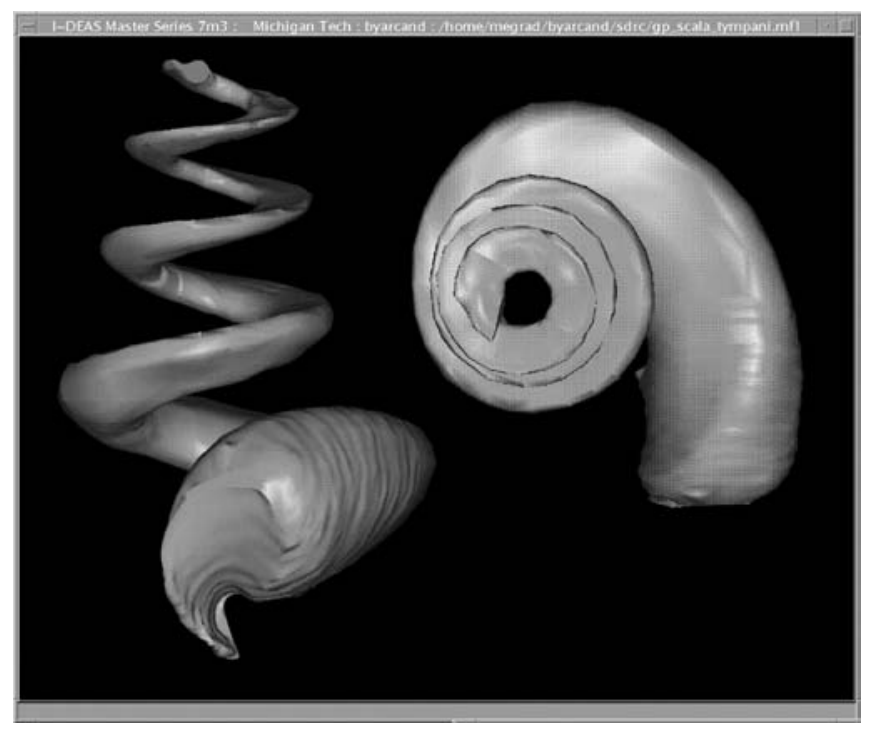

Fig. 3. Solid model of the guinea pig scala tympani assume a position where the electrodes will face inwards toward the modiolus so that the normal of each stimulating site is perpendicular to the cochlea's spiral axis (Fig. 4).

The cochlear spiral was found to have a linear progression along its axis so that bending in that direction is unnecessary. Therefore actuation is required only to adjust the bending curvature about the electrode face of the probe. To accomplish this, actuation chambers are mounted to the backside of the probe (the face opposite the electrodes) and along the entire length. The base chamber that mounts directly to the probe runs the entire $10 \mathrm{~mm}$ length and features a diameter change to fit the probe's tapered profile. For additional localized control of curvature, additional chambers are stacked onto the first. These stacked chambers run from the basil end to some length short of the chamber upon which it is mounted. In this way, the base chamber provides actuation for the entire length of the device while the additional chambers stacked on top provide for supplementary actuation as the insertion proceeds. Elastic spring back in the material provides the necessary force to return the device back to the original perimodiolar shape after the insertion procedure is complete and fluid pressure is removed.

\section{3}

\section{Fabrication process}

The chambers of the actuation device are fabricated from commercially available PET (polyethylene teraphthalate) tubing. PET provides an ideal material to form the actuation chambers because of its ability to be formed through thermal processes and its stiff elastic properties

$(\mathrm{E}=2.7 \mathrm{GPa})$. If the actuation chambers were manufactured of a material that was overly elastic, ballooning would occur instead of the desired change in shape of the cross-section, too stiff and excessive pressures would be required for actuation. PET is also a material that has been widely accepted for use in various medical devices.

The tubing has a diameter of $200 \pm 25 \mu \mathrm{m}$ and a wall thickness of $4 \pm 2 \mu \mathrm{m}$. Processing begins with fabrication (Fig. 5a,b) of the base chamber that runs the full $10 \mathrm{~mm}$ of the device. A $15 \mathrm{~mm}$ length of the PET is cut and $4 \mathrm{~mm}$ of

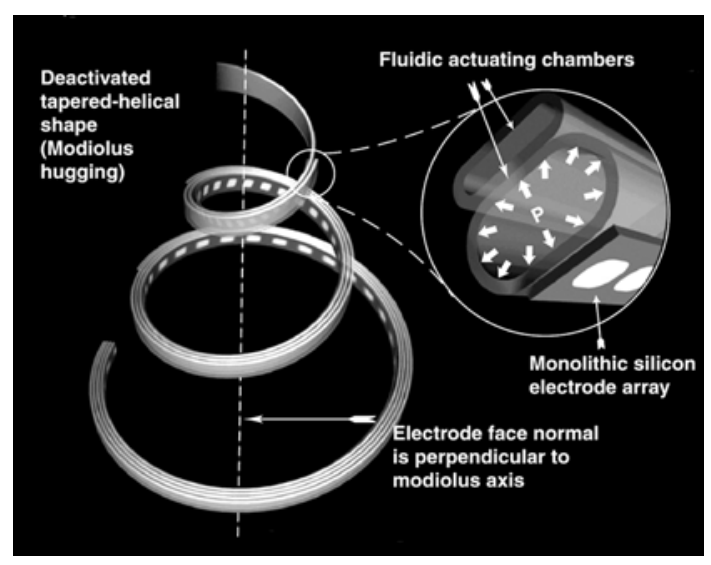

Fig. 4. Design of the articulated perimodiolar cochlear implant. The electrodes on the silicon array face inwards towards the modiolus for optimal nerve stimulation 


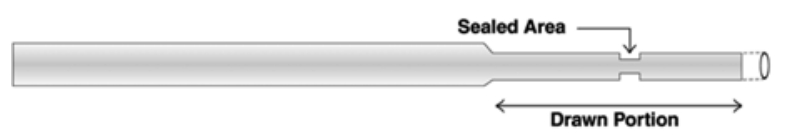

(b) Chamber/Array Assembly (top view)

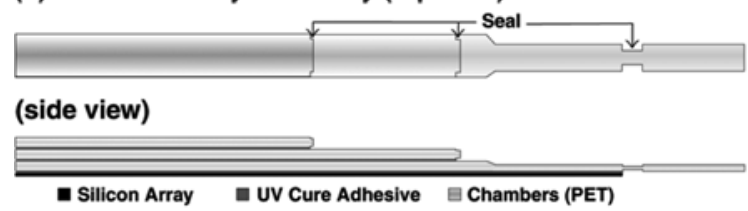

(c) Winding Mandrel

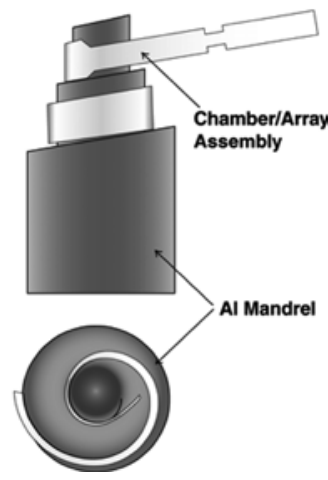

Fig. 5a-c. Fabrication method for an actuating cochlear implant tool. a The base chamber is manufactured; $\mathbf{b}$ additional chambers are fabricated and attached to the base chamber along with the electrode array; $c$ the assembly is wound on the forming mandrel to give it a perimodiolar shape one end is thermally drawn to a length of $8 \mathrm{~mm}$. Drawing reduces the diameter of the leading tip portion of the tubing to $140 \mu \mathrm{m}$ and creates a dual diameter chamber. Next, the tube is placed between two glass plates, clamped and heated to $100{ }^{\circ} \mathrm{C}$ to produce the high-aspect ratio cross-section. Then, the tip portion of the tubing is heat sealed using a specially designed apparatus. The chambers to be stacked on the first are created in a similar fashion, although depending on their length, drawing may be unnecessary. Also on the stacked chambers, any tubing beyond the heat sealed portion is trimmed. The face of each chamber is then roughened slightly with a very fine grit sanding cloth and cleaned with isopropyl alcohol to facilitate bonding. A thin coat of UV cure adhesive (Dymax 201-CTH) is then applied using a low-pressure slotted fixture designed for the purpose. The individual chambers are carefully affixed to each other and placed in a UV light chamber to cure for $5 \mathrm{~min}$. The silicon electrode array is then held flat in a vacuum fixture while the chamber assembly is bonded to it with UV cure adhesive. The device is then carefully clamped and wound around a tapered helical mandrel (electrode array against the mandrel) to form it into the perimodiolar shape (Fig. 5c). The mandrel is micromilled from aluminum and has a very thin coating of silicone applied to the surface to provide protection for the delicate electrode array. Gentle heating in an oven to $110-120{ }^{\circ} \mathrm{C}$ allows the PET chambers to thermally form and fit the mandrel locking the device into a perimodiolar shape, thus building in the residual bending moment referred to in Eq. (1). The device is then released from the mandrel and a pressure source is introduced by means of $183 \mu \mathrm{m}$ OD steel tubing which is inserted into the PET chamber ends for a length of $1 \mathrm{~mm}$. Figure 6a shows a completed three-chambered device without the silicon array while Fig. $6 \mathrm{~b}$ illustrates a device with the silicon array attached.

\section{4}

\section{Testing and modeling}

For testing of actuation potentials, the three-chambered insertion tool prototypes were attached to a pressurized air source. Each chamber was regulated individually using common in-line diaphragm type pressure regulators. To better quantify the actuation potential, a chamber with a constant curling radius was created and measurements were taken of the increase in curling radius with applica-

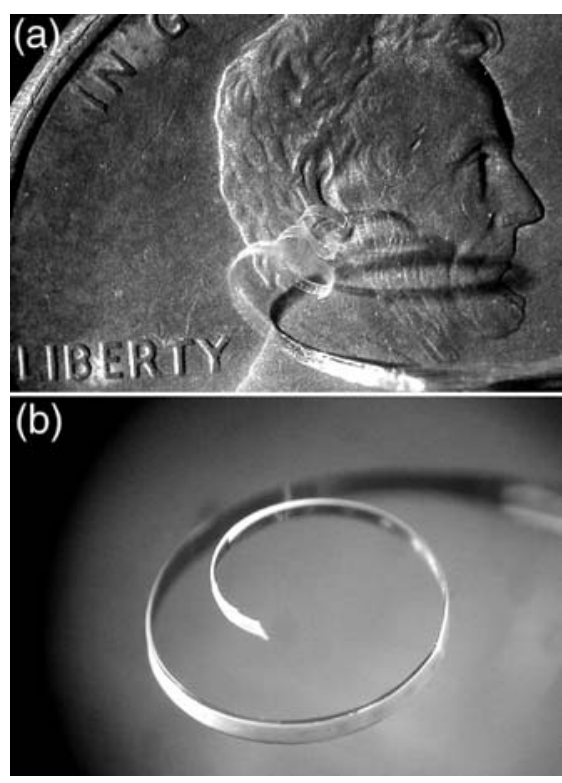

Fig. 6a, b. a A three-chambered actuation tool; b an actuation tool with attached silicon array

tion of pressure (Fig. 7). Figure 8 shows the experimental measurements against predicted values using FEA analysis coupled with Eq. (1). The FEA analysis predicted how the cross-section changed with applied pressure. The crosssection of the manufactured chamber was measured using white light interferometry (Fig. 9b) and modeled in ANSYS 6.0 as a beam using non-linear analysis for large

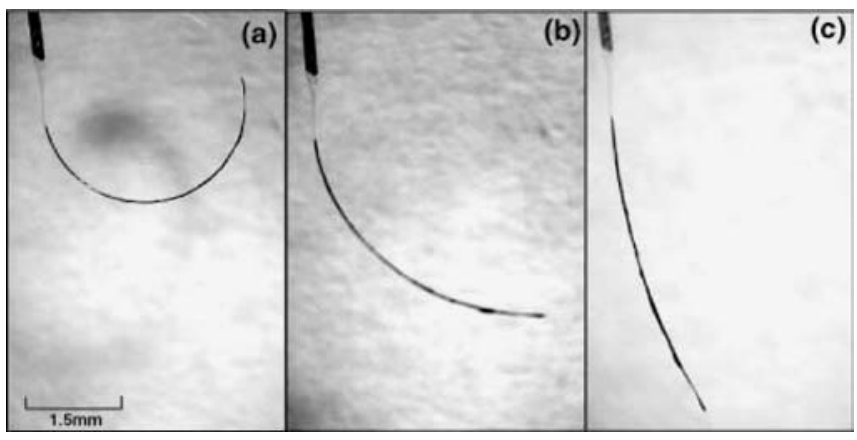

Fig. 7a-c. Actuation of a single chamber with a constant curling radius. Frames a-c show 0, 103, and $206 \mathrm{KPa}$ applied fluidic pressure respectively 


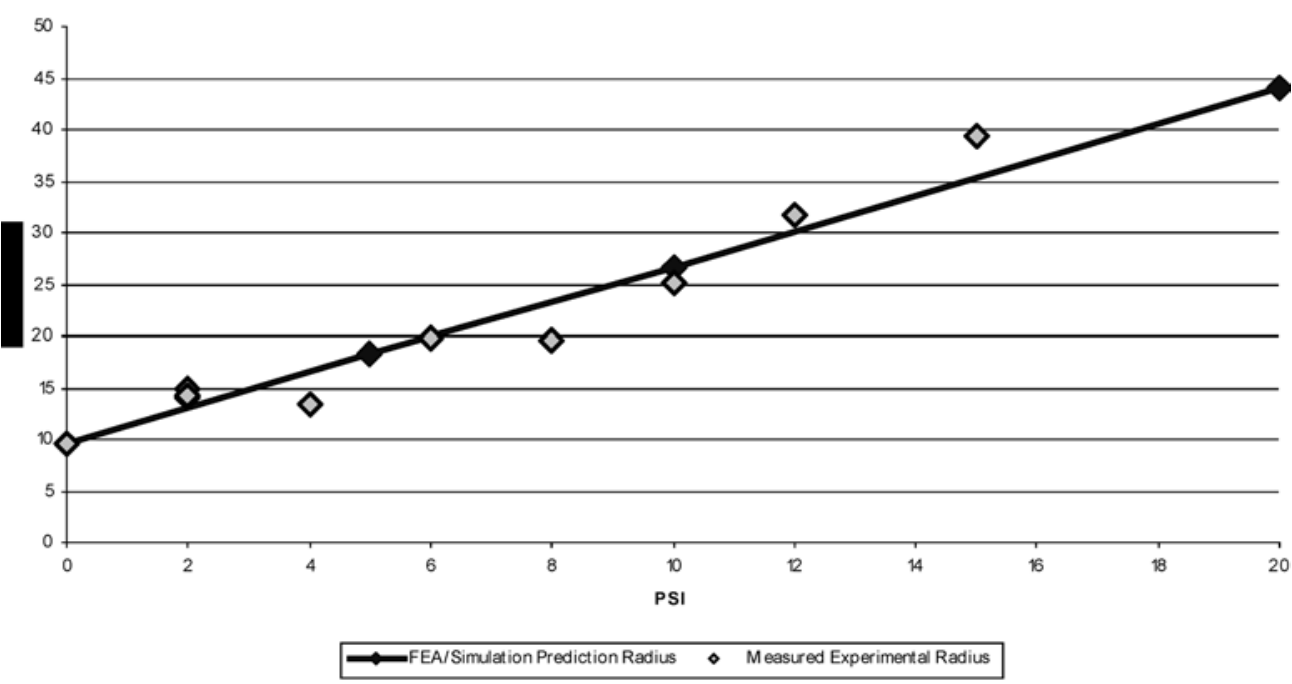

Fig. 8. Results for curling radius vs pressure using FEA analysis to predict chamber cross-section changes and Eq. (1) to estimate the curling radius. Experimentally measured results are also shown deflections (Fig. 9a). The FEA results were then used to calculate the second moment of area of the cross-section about its major axis. This was then applied to Eq. (1) to predict a curling radius change. The predicted results matched within an R-squared value of 0.9375 with those values measured experimentally. The FEA also revealed that the second moment of area increases almost linearally with pressure when the pressures are small when compared to pressures required to produce an almost round cross-section. The experiments exposed that spring back of the material is lost as pressures increase. This is likely due to the high strains seen at key points such as the crimped edges of the chamber. Overall, actuations for curling radius increases of $400 \%$ are easily achieved. By contrast, for the insertion of the device into the guinea pig cochlea, a maximum curling radius increase of only $280 \%$ is required.
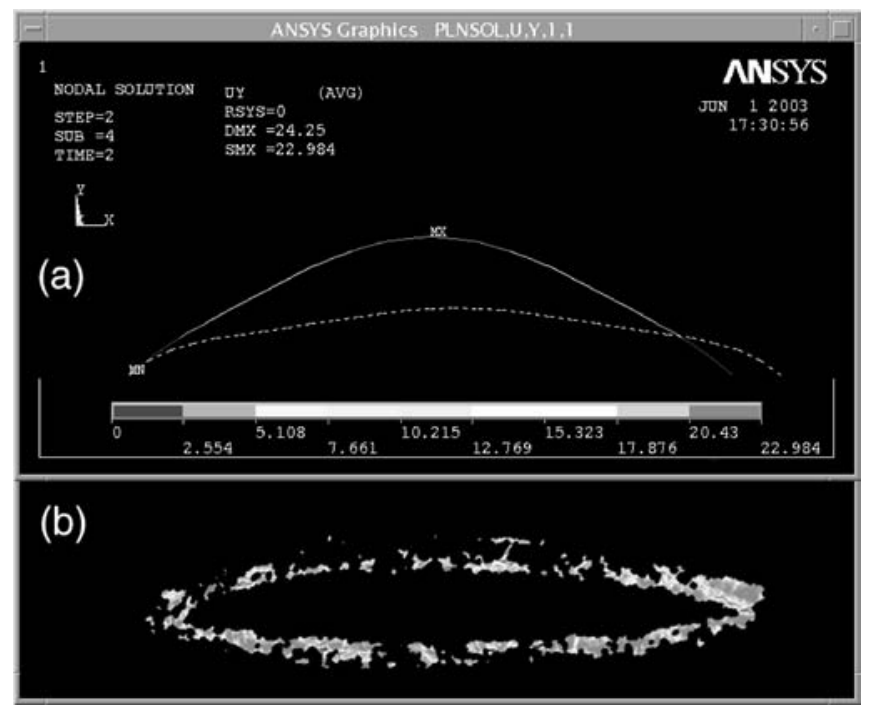

Fig. 9a, b. FEA model result for pressurization of a chamber cross-section a and the measured cross-section from a chamber using white light interferometry $\mathbf{b}$
For purposes of optimizing the number and dimensions of actuation chambers used in the device, a simulation was developed to estimate how well a proposed device could conform to the cochlea during the insertion. The program takes a set of given parameters for the number of chambers in the stack and the dimensions of each. It then represents the cochlear spiral and unactuated device curvature as a discretized curve of varying curling radius. The simulated tool is stepped into the cochlea in $50 \mu \mathrm{m}$ increments while the program compares the radius of the inserted portion of the tool to that of the corresponding portion of the cochlea. The pressure for each chamber inserted in the cochlea is found by minimization of the RMS error between the cochlear curve and the modified device curve using a Runge-Kutta optimization search scheme. Modification of the device curve is found by representing pressure changes in each chamber as shape changes in the cross-section. Calculations of the chamber cross-sections are simplified by representing them as ellipses with changing aspect ratios but constant perimeter values. This way, the second moment of area can be calculated without difficulty and applied back into Eq. (1) to find the modified device curling curvature. Since the device increases in cross-section size as it is pressurized, a check is performed within the program to be sure the device is not exceeding the size of the scala tympani. Figure 10 shows the RMS position error for a simulated three-chambered device insertion. The first two peaks correspond to the second and third chambers entering the cochlea respectively. The last peak indicates the device nearing full insertion when its shape will exactly match that of the cochlea. The simulation demonstrates the positioning error can be kept within $160 \mu \mathrm{m}$ by using as few as three chambers.

\section{5}

\section{Conclusions}

A novel approach to improve the position control and performance of cochlear implants is described here. Benefits of adding fluidic actuators to an implant include greater insertion depths for a larger range of frequency 


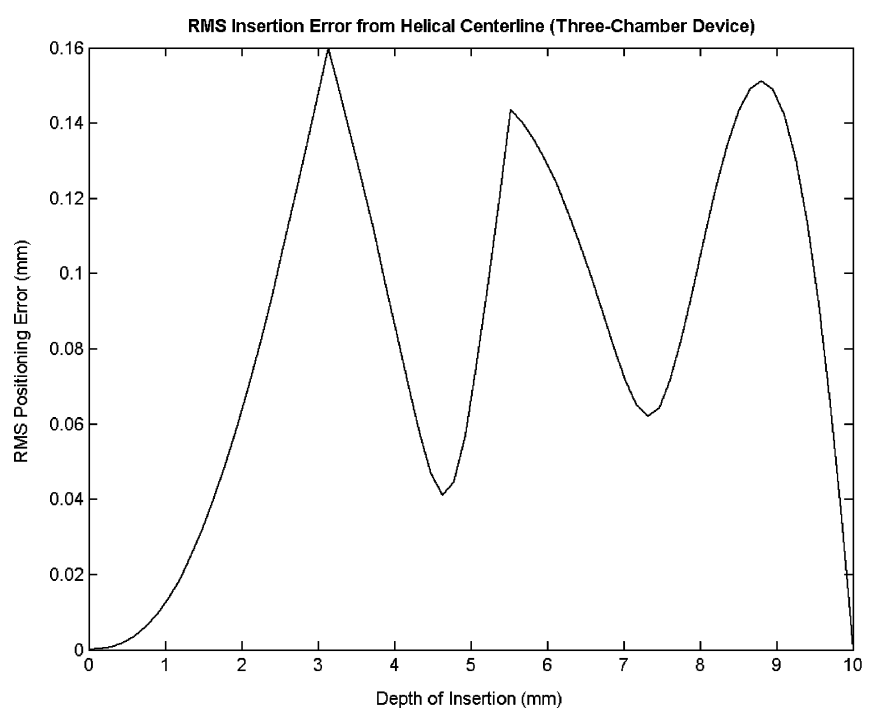

Fig. 10. Simulation results for estimated RMS position error (from ideal) for an optimized three-chambered device during insertion. The peaks indicate when additional chambers along the tool enter the cochlea and begin actuation

perception in an implant recipient and increased proximity of electrodes to stimulating sites. A method for the design and fabrication of fluidic actuators has been demonstrated as well as results of tests with manufactured prototypes. Devices consisting of three actuating chambers show significant radial actuation changes of over $400 \%$.

Future developments include coupling built in strain gages and contact sensors to the pressure regulators to create a feedback control system for insertion. In addition, in vivo guinea pig testing of the device will be performed to characterize actual performance during a cochlear implant procedure.

\section{References}

1. Chen JM; Farb F; Hanusaik L; Shipp D; Nedzelski JM (1999) Depth and quality of electrode insertion. Am J Otology 20(2): 192-197

2. Frijns JHM; Briaire JJ; Grote JJ (2001) The importance of human cochlear anatomy for the results of modiolus-hugging multichannel cochlear implants. Otology Neurotology 22: 340349

3. Shepard RK; Hatsushika S; Clark GM (1993) Electrical Stimulation of the auditory nerve: the effect of electrode position on neural excitation. Hear Res 66: 108-122

4. Brummer SB; Turner MJ (1977) Electrochemical considerations for safe electrical stimulation of the nervous system with platinum electrodes. IEEE Trans Biomed Eng 24: 59-62

5. Kennedy DW (1987) Multichannel intracochlear electrodes: mechanism of insertion trauma. Laryngoscope 97: 42-49

6. Timoshenko SP; Gere JM (1961) Theory of Elastic Stability. McGraw-Hill, New York pp. 278-282

7. Voie AH; Spelman FA (1995) Three-dimensional reconstruction of the cochlea from two-dimensional images of optical sections. Comput Medical Imaging Graph 19(5): 377-384 